\title{
Diseño e Implementación de Sensores Autónomos Inalámbricos para Sistemas Eléctricos de Protección Utilizando Microprocesadores de Bajo Consumo de Potencia
}

\author{
Barahona, Andrés $^{1}$ (D); Salazar, Jaime ${ }^{1}$ iD ; Soria, Mauricio1 $^{\text {(D) }}$; Novoa, Edison ${ }^{1}$ (D) Caina, Darwin² ${ }^{\text {iD }}$; \\ Pérez, Fabián ${ }^{1, *}$ id \\ 'Escuela Politécnica Nacional, Facultad de Ingeniería Eléctrica y Electrónica, Quito, Ecuador \\ ${ }^{2}$ Universidad Central del Ecuador, Facultad de Ciencias, Quito, Ecuador
}

\begin{abstract}
Resumen: Este artículo presenta el diseño de un sensor autónomo para sistemas de protección eléctrica empleando tecnologías innovadoras que, para su operación, se abastecen de recursos energéticos del medio ambiente y además requieren de un bajo consumo de potencia. El prototipo posee tarjetas electrónicas que operan en cuatro etapas. La primera etapa se encarga de adquirir datos reales de corriente de un sistema eléctrico de prueba. En la segunda etapa, mediante el preprocesamiento y análisis de datos, se detectan perturbaciones en la señal de corriente del sistema eléctrico de prueba. La tercera etapa comprende la comunicación de datos por radiofrecuencia, la cual se ejecuta solo cuando su algoritmo de procesamiento ha detectado valores de corriente anormales, caso contrario, la transmisión de información se mantiene deshabilitada y con el dispositivo operando en modo de ultra bajo consumo de energía. La última etapa del sensor autónomo se encarga de proveerlo de energía mediante baterías de litio-polímero y paneles fotovoltaicos que aprovechan el uso y almacenamiento de la energía solar. Finalmente, para probar el sensor diseñado, se realizan varias pruebas de funcionamiento y se analizan los resultados obtenidos.
\end{abstract}

Palabras clave: Sensor autónomo inalámbrico, protecciones eléctricas, energía solar, dispositivos de ultra bajo consumo de potencia, comunicación con XBee S1.

\section{Design and Implementation of Autonomous Wireless Sensors for Electrical Protection Systems Using Ultra Low Power Consumption Microprocessors}

\begin{abstract}
This article constitutes the design of an autonomous sensor for electrical protection systems using new technologies that includes energy harvesting and the low power consumption of the electronic elements used. The prototype is developed in four stages. The first stage focuses on acquiring information from an external electrical system using a current sensor, that data is processed in the second stage by the development card which detects when a disturbance occurs in the current signal of the external electrical system and prioritizes the low power consumption of the devices when it is not necessary to transmit information. The third stage covers radio frequency data communication, which is executed once the processing algorithm has detected that the current exceeds the limits of normal operation, otherwise the transmission of information is disabled, and the device is in a low consumption mode. The last stage makes energy harvesting and storage of it, using solar modules, the solar radiation is harvested and transformed into electrical energy, the same that feeds the prototype, the excess energy is stored in a lithium-polymer battery, which works in absence of solar radiation. Finally, the equipment is integrated in a printed circuit board that constitutes the final prototype. To verify the correct operation of the designed sensor, many operation tests are carried out and the results obtained are analyzed.
\end{abstract}

Keywords: Wireless autonomous sensor, electrical protection, solar energy, ultra-low power consumption device, XBee S1 communication modules.

\section{INTRODUCCIÓN}

Dentro de un sistema eléctrico los transformadores de medida cumplen la función de reducir el voltaje y la corriente a niveles estándar para que el manejo de las señales eléctricas sea seguro. Estas señales permiten operar los relés de protección y brindan información a los dispositivos de medición. En el devanado secundario de los Transformadores de Corriente (TCs) se genera una corriente proporcional y en fase a la corriente del primario, la misma que está normalizada en 1,2

*fabian.perez@epn.edu.ec

Recibido: 21/08/2020

Aceptado: 06/07/2021

Publicado: 31/08/2021

$10.33333 /$ rp.vol48n1.03

CC 4.0 
o 5 amperios. En el secundario de los Transformadores de Potencial (TPs) se tiene un voltaje proporcional al primario y está estandarizado en 120 Voltios (Ramirez, 2014).

En una subestación, las protecciones eléctricas se energizan por medio de fuentes de energía que cuentan con distintos puntos para su alimentación dentro de los llamados servicios auxiliares, entre estos están: el trasformador de distribución, el terciario de un transformador de potencia, generador de emergencia o bancos de baterías (Ochoa, 2013).

Los relés de protección supervisan las señales eléctricas de un sistema en el que la información se adquiere, desde los instrumentos de medida, por medios cableados (Ochoa, 2013).

Los antecedentes mencionados implican un costo económico, un consumo energético, un espacio físico y obras civiles costosas para el cableado e implementación de los sistemas eléctricos de medición y protección. Actualmente, está en auge el uso de dispositivos de procesamiento y transmisión inalámbrica de datos con ultra bajo consumo energético puesto que permiten hacer más eficientes a los sistemas eléctricos (Caina, 2019).

A continuación, se describe el diseño e implementación de un prototipo de sensor autónomo inalámbrico de corriente que podrá utilizarse en redes eléctricas donde los tiempos de respuesta de los componentes del prototipo sean compatibles con el normal funcionamiento de los sistemas de protección eléctrica.

\section{DISEÑO E IMPLEMENTACIÓN}

El diseño del sensor se encuentra dividido en subsistemas, los mismos que se encuentran detallados en la Tabla 1 junto con sus respectivas funciones.

Tabla 1. Subsistemas del diseño del prototipo

\begin{tabular}{|c|c|}
\hline Subsistemas & Funciones \\
\hline $\begin{array}{l}\text { Adquisición de } \\
\text { datos }\end{array}$ & $\begin{array}{l}\text { Utilizando un sensor de corriente, se } \\
\text { obtiene la información del sistema } \\
\text { eléctrico externo. }\end{array}$ \\
\hline $\begin{array}{l}\text { Procesamiento de la } \\
\text { información }\end{array}$ & $\begin{array}{l}\text { Evaluar las mediciones de corriente del } \\
\text { sistema eléctrico externo para detectar } \\
\text { perturbaciones en la señal. } \\
\text { Priorizar el consumo de potencia durante } \\
\text { la operación del prototipo. }\end{array}$ \\
\hline $\begin{array}{l}\text { Transmisión } \\
\text { inalámbrica de } \\
\quad \text { datos }\end{array}$ & $\begin{array}{l}\text { La transmisión inalámbrica de datos } \\
\text { hacia el receptor inicia cuando se } \\
\text { presenten valores atípicos en la onda de } \\
\text { corriente. }\end{array}$ \\
\hline $\begin{array}{l}\text { Recolección de } \\
\text { energía solar }\end{array}$ & $\begin{array}{l}\text { Captar la radiación solar y convertirla en } \\
\text { energía eléctrica. } \\
\text { Adecuar el voltaje requerido para cargar } \\
\text { la batería. }\end{array}$ \\
\hline $\begin{array}{l}\text { Almacenamiento de } \\
\text { energía eléctrica }\end{array}$ & $\begin{array}{l}\text { La energía eléctrica se recolecta en una } \\
\text { batería que energiza al prototipo en } \\
\text { ausencia de radiación solar. }\end{array}$ \\
\hline
\end{tabular}

\subsection{Adquisición de datos}

Se recolecta información de un sistema eléctrico de prueba mediante un medidor de corriente que genera una señal de salida analógica de voltaje proporcional al valor de la corriente medida. El microcontrolador (MCU) evalúa los datos para determinar si el sistema eléctrico externo opera dentro de los límites normales de corriente.

\section{- $\quad$ Sensor de corriente de efecto Hall}

La corriente de operación del sistema eléctrico externo es superior a la corriente del sensor autónomo, debido a esto los sistemas se encuentran eléctricamente aislados, para lo cual se utiliza el sensor de efecto Hall LTS 15-NP que proporciona una salida instantánea con gran precisión, alta inmunidad a interferencias y un ancho de banda de $100 \mathrm{kHz}$. El sensor tiene configuraciones para medir corrientes de 5A, 7,5A, 15A (LEM, 2017).

Para el prototipo el ancho de banda corresponde también a los $100 \mathrm{kHz}$; valor notablemente superior al de los transformadores de corriente tradicionales.

\section{- $\quad$ Diodo Zener}

Para proteger el sensor de corriente de sobrevoltajes generados por los paneles fotovoltaicos se utiliza el diodo Zener 1N4733, el cual actúa cuando el voltaje supera los 5,1V (VISHAY, 2003). En la Figura 1, se muestra el circuito implementado.

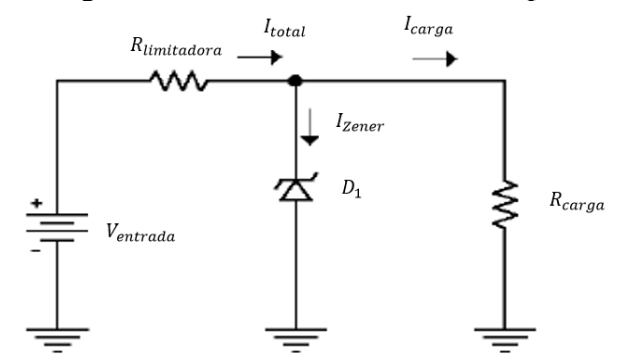

Figura 1. Circuito implementado para el diodo Zener (Mijarez, 2014)

El exceso de voltaje se disipa utilizando una resistencia limitadora, la misma que se calcula con la Ecuación (1) (Mijarez, 2014), para lo cual se utiliza el valor máximo de voltaje generado por los paneles fotovoltaicos y la menor corriente que fluye en el diodo Zener.

$$
R_{\text {lim }}=\frac{V_{\text {entrada }}-V_{D 1}}{I_{\text {Zener }}+I_{\text {carga }}}
$$

$V_{\text {entrada }}$ : voltaje de la fuente $(\mathrm{V}), V_{D 1}$ : voltaje en el diodo Zener (V), I Zener: corriente del diodo Zener (A), I $I_{\text {carga }}$ : corriente del sensor (A), $R_{\text {lim }}$ : resistencia limitadora $(\Omega)$.

$$
R_{\lim }=\frac{6 V-5,1 V}{(28+1) m A}=\frac{0,9 V}{29 m A}=31 \Omega \Rightarrow R_{\lim }=33 \Omega
$$

\subsection{Procesamiento de la información}

El elemento inteligente del prototipo lo conforma la tarjeta MSP430-EXPFR5969, la cual es muy utilizada en aplicaciones que requieren muy bajo consumo de potencia (Texas Instruments, 2015). La tarjeta incluye un conversor analógico-digital (ADC) que se utiliza para adquirir la señal entregada por el sensor de corriente y el voltaje generado por los módulos solares. Además, la tarjeta cuenta con un módulo de comunicación serial asincrónica - UART que se utiliza para transmitir la información al dispositivo emisor de 
comunicación por radiofrecuencia cuando se detecta anomalías en la señal de corriente del sistema eléctrico de prueba.

La tarjeta de desarrollo posee modos de operación con diferentes niveles de consumo en los que se desactivan funciones del microcontrolador cuando no se las utiliza (Texas Instruments, 2017). Por ejemplo, en el prototipo desarrollado el microcontrolador deja su modo de bajo consumo mientras recibe y evalúa los datos que proporciona el sensor de corriente.

En la Figura 2, se detalla el diagrama de flujo del algoritmo implementado, en el que destacan las siguientes funciones: adquirir 20 muestras por cada ciclo de la señal de corriente; almacenar la información de los últimos dos ciclos; detectar perturbaciones en la señal; determinar valores máximos y medir el valor eficaz de corriente del sistema eléctrico de prueba; determinar cuándo se realiza la transmisión de información y habilitar la carga de la batería cuando se disponga del voltaje suficiente en los módulos solares.

\subsection{Transmisión inalámbrica de datos}

Se utiliza módulos XBee Serie 1, debido a sus esquemas de bajo consumo de energía, además que permiten formar redes de sensores con una comunicación confiable (DIGI, 2018). La transmisión inalámbrica de datos se realiza entre el prototipo y el computador que, para este caso, simulará la operación de un relé de protección. Los módulos de comunicación XBee S1 se configuran para trabajar en el modo de bajo consumo, llamado sueño ligero, ya que el mismo dispone de 2,6 ms para despertar y su consumo de corriente es menor a $50 \mu \mathrm{A}$ (DIGI, 2018).

\section{- Configuración de la tarjeta XBee S1}

La configuración para establecer comunicación entre los módulos de radiofrecuencia se realiza utilizando el software XCTU. En la Tabla 2, se especifica la configuración de las variables en cada uno de los módulos.

Tabla 2. Parámetros modificados utilizando XCTU

\begin{tabular}{ccc}
\hline Parámetros & XBee emisor & XBee receptor \\
\hline PAN ID & 9032 & 9032 \\
DH & 0 & 0 \\
DL & 1 & 0 \\
MY & 0 & 1 \\
CE & 0 & 0 \\
PL & 4 & 4 \\
SM & 2 & 2 \\
BD & 115200 & 115200 \\
Bits de datos & 8 & 8 \\
Paridad & Ninguna & Ninguna \\
Bits de parada & 1 & 1 \\
Control de flujo & Ninguno & Ninguno
\end{tabular}

\subsection{Recolección de energía solar}

Con la tecnología actual se puede recolectar energía proveniente de distintas fuentes existentes en el medio ambiente; la cantidad de potencia por unidad de área que se genera en cada caso es distinta y se resume en la Tabla 3.

Tabla 3. Energía recolectada de distintas fuentes (Catalán López, 2013)

\begin{tabular}{ccc}
\hline Tipo de Energía & Fuente de energía & Potencia generada \\
\hline \multirow{2}{*}{ Solar } & $\begin{array}{c}\text { Interior } \\
\text { Exterior }\end{array}$ & $\begin{array}{c}10 \mu \mathrm{W} / \mathrm{cm}^{2} \\
10 \mathrm{~mW} / \mathrm{cm}^{2}\end{array}$ \\
\hline \multirow{2}{*}{ Térmica } & $\begin{array}{c}\text { Humano } \\
\text { Industria }\end{array}$ & $\begin{array}{c}25 \mu \mathrm{W} / \mathrm{cm}^{2} \\
1-10 \mathrm{~mW} / \mathrm{cm}^{2}\end{array}$ \\
\hline \multirow{2}{*}{ Mecánica } & Humano & $4 \mu \mathrm{W} / \mathrm{cm}^{2}$ \\
& Industria & $100 \mu \mathrm{W} / \mathrm{cm}^{2}$ \\
\hline \multirow{2}{*}{ Radiofrecuencia } & $\begin{array}{c}\text { Comunicación } \\
\text { (GSM) }\end{array}$ & $0,1 \mu \mathrm{W} / \mathrm{cm}^{2}$ \\
& Wi-Fi & $1 \mu \mathrm{W} / \mathrm{cm}^{2}$ \\
\hline
\end{tabular}

Para la implementación del prototipo se seleccionó la energía solar, ya que se puede recolectar mayor cantidad de potencia, en instalaciones exteriores, en comparación con los otros tipos de energía mencionados en la Tabla 3.

\section{- Estimación del consumo de energía}

Para dimensionar el número de módulos fotovoltaicos se necesita estimar el consumo de potencia de todos los elementos que constituyen el prototipo, para ello se consideró dos estados de operación, cuando transmite y cuando no transmite información, como se detalla en la Tabla 4.

Tabla 4. Consumo de potencia del sensor autónomo

\begin{tabular}{ccc}
\hline Elemento & $\begin{array}{c}\text { Consumo sin } \\
\text { transmisión de } \\
\text { datos }(\mathbf{m W})\end{array}$ & $\begin{array}{c}\text { Consumo con } \\
\text { transmisión de } \\
\text { datos }(\mathbf{m W})\end{array}$ \\
\hline $\begin{array}{c}\text { Sensor de corriente } \\
\text { Microcontrolador }\end{array}$ & 115 & 115 \\
MSP430FR-5669 & 12,5 & 13,3 \\
XBee S1 & 0,13 & 165,6 \\
\hline Total & 128 & 294 \\
\hline
\end{tabular}

Posterior a lo mencionado, se calcula el consumo de energía diario del prototipo de acuerdo con el tiempo de operación en cada uno de sus estados, como lo muestra la Tabla 5.

Tabla 5. Energía diaria consumida

\begin{tabular}{|c|c|c|c|c|}
\hline Estado & $\begin{array}{c}\text { Potencia } \\
\text { (W) }\end{array}$ & $\begin{array}{c}\text { Tiempo de } \\
\text { funcionamiento } \\
\text { al día }\end{array}$ & $\begin{array}{l}\text { Energía } \\
\text { total } \\
\text { necesaria } \\
\text { (Wh/día) }\end{array}$ & $\begin{array}{c}\text { Energía } \\
\text { total (con } \\
\text { margen del } \\
25 \%) \\
\text { (Wh/día) }\end{array}$ \\
\hline $\begin{array}{c}\text { Sin } \\
\text { transmisión } \\
\text { de } \\
\text { información }\end{array}$ & 0,1536 & $\begin{array}{c}23 \text { horas } 45 \\
\text { minutos }\end{array}$ & 3,648 & 4,56 \\
\hline $\begin{array}{c}\text { Con } \\
\text { transmisión } \\
\text { de } \\
\text { información } \\
\end{array}$ & 0,3528 & 15 minutos & 0,0882 & 0,11 \\
\hline
\end{tabular}

La energía total se ha calculado con un $25 \%$ de energía de reserva adicional a fin de superar algún imprevisto meteorológico que afecte la recolección del recurso solar. El porcentaje de reserva de energía podría variar según el sitio de instalación del prototipo y en algunos casos podría no requerirse. 


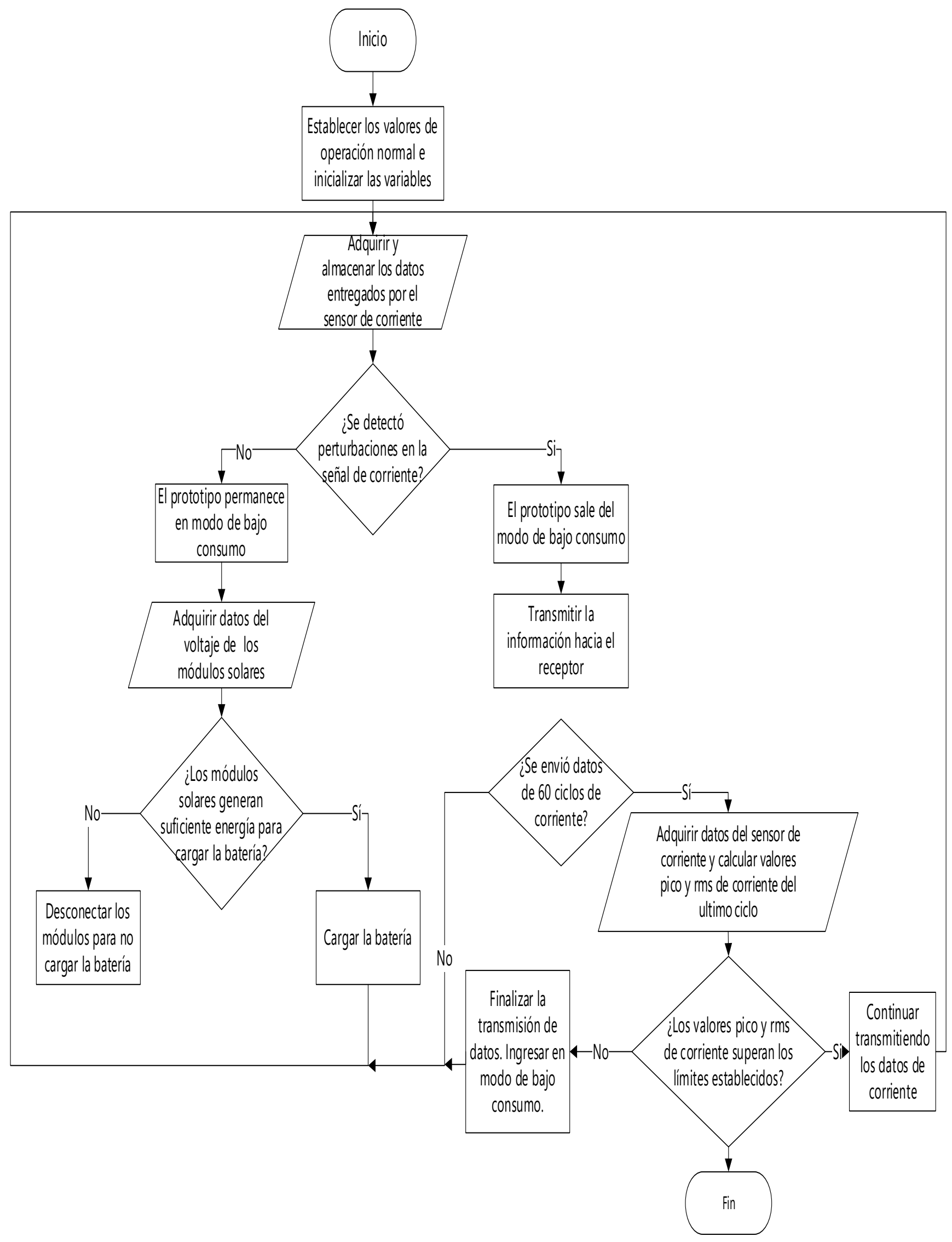

Figura 2. Diagrama de flujo del algoritmo de procesamiento de datos 
El tiempo de funcionamiento para cada uno de los estados se estableció considerando que la información se transmite solo cuando aparecen perturbaciones en la onda de corriente. La mayoría del tiempo no se transmite información.

Se calcula el consumo diario promedio de las cargas DC sumando los consumos de energía cuando el prototipo transmite datos y también cuando no lo hace, como se muestra en la Ecuación (2) (Alonso, 2016).

$$
\begin{gathered}
E_{\text {cmd } D C}=E_{\text {transmitiendo }}+E_{\text {No transmitiendo }} \\
E_{c m d D C}=(4,56+0,11) W h / \text { día }=4,67 \mathrm{Wh} / \text { día }
\end{gathered}
$$

Para la determinación del número de módulos fotovoltaicos se considera una eficiencia teórica del $95 \%$ en los elementos de almacenamiento y un rendimiento del $100 \%$ en los conductores. Con la Ecuación (3) se obtiene el consumo diario promedio de energía (Alonso, 2016).

$$
E_{c m d}=\frac{E_{\text {cmd } D C}+\frac{E_{c m d A C}}{\eta_{\text {inversor }}}}{\eta_{\text {bateria }} \times \eta_{\text {conductor }}}
$$

$E_{c m d}$ : consumo diario promedio de energía $(W h / d i ́ a)$, $E_{c m d D C}$ : consumo diario promedio de cargas que consumen energía continua $(W h / d i ́ a), E_{c m d A C}$ : consumo diario promedio de cargas que consumen energía alterna $(W h /$ día $)$, $\eta_{\text {inversor }}$ : rendimiento del inversor, $\eta_{\text {bateria }}$ : eficiencia de la batería, $\eta_{\text {conductor }}$ : eficiencia de los conductores.

El prototipo no cuenta con cargas $\mathrm{AC}$, por tal motivo las componentes $E_{\text {cmd AC }}$ y $\eta_{\text {inversor }}$ no se consideran en el cálculo. Reescribiendo la Ecuación (3), se tiene:

$$
E_{\text {cmd }}=\frac{E_{c m d, D C}}{\eta_{\text {bateria }} \times \eta_{\text {conductor }}}=\frac{4,67 \mathrm{Wh} / \text { día }}{0,95 \times 1}=4,92 \mathrm{Wh} / \text { día }
$$

Con el valor del consumo diario promedio de energía se calcula la energía total anual $\left(E_{T}\right)$ que consume el prototipo, como se muestra en la Ecuación (4) (Alonso, 2016):

$$
E_{T}=E_{c m d} \times 365 \text { días }
$$

$E_{T}=4,92 \times 365=1795,8 W h / a \tilde{n} o=1,7958 \mathrm{kWh} / a \tilde{\text { ñ } o}$

El valor total de energía anual permite conocer el consumo energético anual del prototipo y compararlo con el consumo que podría demandar de la red eléctrica, como se indica en la Ecuación (5) (Alonso, 2016). Conociendo que el costo del $\mathrm{kWh}$ en Quito, en bajo voltaje, es de 0,083USD, se puede analizar el ahorro obtenido al implementar el prototipo (Agencia de Regulación y Control de Electricidad, 2019).

$$
\begin{gathered}
C_{\text {anual }}=E_{T} \times \text { tarifa } \\
C_{\text {anual }}=1,7958 \mathrm{kWh} / \text { año } \times 0,083 \text { USD } / \mathrm{kWh} \\
C_{\text {anual }}=0,15 \text { USD } / \text { año }
\end{gathered}
$$

Cuando el prototipo opera en el estado de bajo consumo, existe una reducción económica y energética aun cuando se encuentra transmitiendo datos. Para comprobarlo se realizan los mismos cálculos, pero ahora se mantiene el estado de transmisión de información las 24 horas, como lo muestra la Tabla 6 .

Tabla 6. Energía consumida diariamente transmitiendo información

\begin{tabular}{ccccc}
\hline Estado & $\begin{array}{c}\text { Potencia } \\
(\mathbf{W})\end{array}$ & $\begin{array}{c}\text { Tiempo de } \\
\text { funcionamiento } \\
\text { al día }\end{array}$ & $\begin{array}{c}\text { Energía } \\
\text { total } \\
\text { necesaria } \\
(\mathbf{W h} / \text { día })\end{array}$ & $\begin{array}{c}\text { Energía } \\
\text { total } \\
\text { necesaria } \\
\text { con margen } \\
\text { de } \\
\text { seguridad } \\
(\mathbf{2 5 \%}) \\
(\mathbf{W h} / \mathbf{d i ́})\end{array}$ \\
\hline $\begin{array}{c}\text { Con } \\
\text { transmisión } \\
\text { de datos }\end{array}$ & 0,3528 & 24 horas & 8,4672 & 10,584 \\
\hline
\end{tabular}

Desarrollando los cálculos explicados anteriormente:

$$
\begin{gathered}
E_{c m d D C 2}=10,584 \mathrm{Wh} / \text { día } \\
E_{c m d 2}=\frac{E_{c m d, D C 2}}{\eta_{\text {bateria }} \times \eta_{\text {conductor }}}=\frac{10,584 \mathrm{Wh} / \text { día }}{0,95 \times 1} \\
E_{c m d 2}=11,141 \mathrm{Wh} / \text { día } \\
E_{T 2}=11,141 \mathrm{Wh} / \text { día } \times 365 \text { días }=4066,5 \mathrm{Wh} / \text { año } \\
E_{T 2}=4,0665 \mathrm{kWh} / \text { año } \\
C_{\text {anual2 }}=4,0665 \mathrm{kWh} / \text { año } \times 0,083 \mathrm{USD} / \mathrm{kWh} \\
C_{\text {anual } 2}=0,34 \mathrm{USD} / \text { año }
\end{gathered}
$$

Se calcula el porcentaje de reducción de energía anual consumida mediante la Ecuación (6) (Rustom, 2012).

$$
\begin{gathered}
P_{\text {reducción }}=\frac{E_{T 2}-E_{T}}{E_{T 2}} \times 100 \% \\
P_{\text {reducción }}=\frac{4,0665-1,7958}{4,0665} \times 100 \%=55,84 \%
\end{gathered}
$$

Al permanecer en modo de bajo consumo se disminuye en un $55,84 \%$ la energía total consumida en un año por el prototipo; lo que a su vez disminuye el costo energético del prototipo.

\section{- Cálculo de la cantidad de módulos solares}

Para dimensionar el número de módulos solares se utiliza el valor de radiación solar, en el lugar que se implementa el prototipo, en este caso Quito-Ecuador. La información se obtiene del Atlas Solar del Ecuador elaborado por la ARCONEL.

El número de módulos fotovoltaicos necesarios para la energización del prototipo se calcula con el valor de radiación 
del mes más crítico, que es el mes de menor radiación solar en el sitio de instalación (Radiación $n_{m e s-c r i ́ t i c o ~}$ (Abella, 2012). Con el valor de radiación crítica se determina la hora solar pico $(H P S)$ que es la unidad que mide la radiación que proviene del sol cuando se tiene una radiación solar hipotética constate de $1000 \mathrm{~W} / \mathrm{m}^{2}$, como se muestra en la Ecuación (7), (Alonso, 2016).

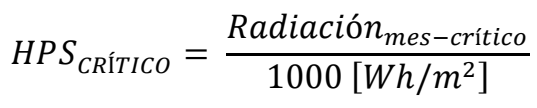

$$
\begin{aligned}
& H P S_{\text {CRÍTICO }}=\frac{4925 \mathrm{Wh} / \mathrm{m}^{2} / \text { día }}{1000 \mathrm{~W} / \mathrm{m}^{2}}=4,925 \mathrm{HPS}
\end{aligned}
$$

De la misma forma se calcula el consumo energético diario en amperio hora $\left(E_{A h}\right)$, el mismo que se obtiene de la Ecuación (8) (Alonso, 2016). Se utilizó baterías de 3,7 V puesto que es el voltaje al que trabajan los elementos del prototipo.

$$
\begin{gathered}
E_{A h}=\frac{E_{c m d}}{V_{b a t}} \\
E_{A h}=\frac{4,92 W h / d i ́ a}{3,7 \mathrm{~V}}=1,33 \mathrm{Ah} / \text { día }
\end{gathered}
$$

Por último, se calculó la corriente generada por los módulos fotovoltaicos en las condiciones del mes crítico $\left(I_{G}\right)$ (Alonso, 2016), la cual se obtiene a través de la Ecuación (9):

$$
\begin{gathered}
I_{G}=\frac{E_{A h}}{H P S_{\text {crítico }}} \\
I_{G}=\frac{1,33 \mathrm{Ah} / \text { día }}{4,925 \mathrm{HPS}}=0,27 \mathrm{~A}
\end{gathered}
$$

Al conectar los módulos fotovoltaicos en paralelo se aumenta la corriente producida (CIRCE, 2015). Este valor se obtiene dividiendo $I_{G}$ para la máxima corriente de los módulos solares $\left(I_{\max }\right)$, la cual es proporcionada por el fabricante y es de 0,2 A (IXYS, 2018). Con la Ecuación (10) se obtiene el número de módulos solares requeridos (Alonso, 2016):

$$
\begin{gathered}
N_{\text {módulos }}=\frac{I_{G}}{I_{\max }} \\
N_{\text {módulos }}=\frac{0,27 \mathrm{~A}}{0,20 \mathrm{~A}}=1,35 \text { módulos } \\
N_{\text {módulos }}=2 \text { módulos }
\end{gathered}
$$

\subsection{Almacenamiento de energía eléctrica}

La energía que se ha recolectado de los módulos fotovoltaicos se almacena en baterías recargables con el objetivo de brindar una autonomía energética al sensor cuando no se tenga radiación solar o ésta sea insuficiente. Dicha autonomía está directamente relacionada con la capacidad de la batería.

\section{Dimensionamiento de la batería}

La capacidad de la batería depende de la cantidad de días que se desee que el prototipo funcione sin recibir energía solar. El tiempo que se ha escogido es de dos días, el mismo que se considera adecuado hasta que los módulos fotovoltaicos vuelvan a captar radiación fotovoltaica. En la Ecuación (11) se calcula la capacidad de la batería (Alonso, 2016):

$$
C_{b a t}=\frac{E_{c m d} \times N}{p_{d e s c} \times V_{b a t}}
$$

$C_{\text {bat }}$ : capacidad de almacenamiento de la batería (Ah), $E_{c m d}$ : consumo diario promedio de energía $(W h /$ día $), N$ : tiempo en días que se desee para autoabastecimiento del prototipo, $p_{\text {desc }}$ : profundidad de descarga de la batería, en las baterías de litio este factor es del $0,9, V_{b a t}$ : voltaje en el que funciona la batería (V).

$$
C_{\text {bat }}=\frac{4,92 W h / \text { día } \times 2}{0,9 \times 3,7 V}=2,954 A h
$$

Se selecciona una batería de 3 Ah debido a que es un equipo fácil de encontrar en el mercado. El material de este acumulador es de litio-polímero ya que presenta algunas características importantes como: una mayor vida útil, posee una descarga lineal, mayores ciclos de carga y descarga, no presenta el efecto memoria, que es el efecto que reduce su capacidad por cargas incompletas y se puede almacenar mayor cantidad de voltaje que en otros tipos de baterías (J\&A, 2012).

Gracias a la Ecuación (10) se obtuvo que se necesitan dos módulos solares en paralelo, pero, además, se debe conocer el tiempo de carga de la batería cuando ésta se encuentre descargada totalmente, este tiempo se lo obtiene mediante la Ecuación (12) (Alonso, 2016):

$$
\text { tiempo }_{\text {carga }}=\frac{C_{\text {bat }}}{I_{\max }}
$$

Con dos módulos solares el tiempo de carga de la batería es:

$$
\text { tiempo }_{\text {carga }}=\frac{3000 \mathrm{mAh}}{(200+200) m A}=7,5 \text { horas }
$$

Este valor de 7,5 h es alto debido a que, si bien se tiene 12 horas hipotéticas de energía solar por día, las que son realmente útiles son de $10 \mathrm{am}$ a $4 \mathrm{pm}$, en total 6 horas. Por lo que se aumenta un módulo fotovoltaico adicional, para que este tiempo de carga disminuya, por lo que con 3 módulos solares se tiene:

$$
\text { tiempo }_{\text {carga }}=\frac{3000 m A h}{(200+200+200) m A}=5 \text { horas }
$$

Se tiene un tiempo de 5 horas para cargar la batería cuando ésta se encuentre totalmente descargada y los módulos solares se encuentren produciendo su corriente nominal de $200 \mathrm{~mA}$. 


\section{- Regulador de carga}

El regulador de carga permite una carga eficiente de la batería y evita que el acumulador de energía opere en situaciones dañinas en las que se ven afectadas sus propiedades físicas.

Para el dimensionamiento y selección del regulador que se ajuste a las necesidades del sensor autónomo se requiere la corriente de entrada máxima que llega al regulador, la Ecuación (13) (Alonso, 2016) permite obtener este valor:

$$
I_{\text {entrada }}=f_{\text {seguridad }} \times I_{\text {max }} \times N_{\text {paralelo }}
$$

$I_{\text {entrada }}$ : entrada de corriente al regulador $(\mathrm{A}), f_{\text {seguridad }}$ : se selecciona un $25 \%$ adicional por seguridad, $I_{\max }$ : corriente nominal a los que funcionan los módulos solares (A), $N_{\text {paralelo }}$ : número de módulos solares conectados en paralelo.

$$
I_{\text {entrada }}=1,25 \times 200 \mathrm{~mA} \times 3=900 \mathrm{~mA}=0,9 \mathrm{~A}
$$

Además, para calcular la corriente de salida del regulador se utiliza la Ecuación (14) (Alonso, 2016):

$$
I_{\text {salida }}=\frac{f_{\text {seguridad }} \times\left(P_{D C}+\frac{P_{A C}}{\eta_{\text {inversor }}}\right)}{V_{\text {batería }}}
$$

$I_{\text {salida }}$ : salida de corriente del regulador $(\mathrm{A}), P_{D C}$ : potencia DC requerida por las cargas $(\mathrm{W}), P_{A C}$ : potencia $A C$ requerida por las cargas (W), $\eta_{\text {inversor }}$ : eficiencia del inversor.

$$
I_{\text {salida }}=\frac{1,25 \times(0,1476 \mathrm{~W}+0,3336 \mathrm{~W})}{3,7 \mathrm{~V}}=0,162 \mathrm{~A}
$$

Se escogió el regulador de carga WeMos D1 Mini TP5410 debido a que es un elemento que se ajusta a las corrientes de entrada y salida calculadas y posee la característica que trabaja de una manera eficiente con las baterías de litio.

\section{- Conversor DC-DC elevador}

La utilización de un conversor DC-DC elevador mejora la autogestión energética del sensor autónomo puesto que, para cuando el voltaje generado por los módulos fotovoltaicos sea menor a $5 \mathrm{~V}$, este elemento eleva el voltaje y lo mantiene constante. Se elige un conversor elevador comercial que cumple con los requerimientos necesarios, el cual tiene la numeración MT3608 (AEROSEMI, 2015).

\section{- Conversor DC-DC reductor}

Se utilizó un conversor DC-DC reductor para ajustar los voltajes a los que opera la tarjeta MSP430-EXPFR5969 y los módulos XBee que es de 3,1 V lo que mejora el bajo consumo de potencia del prototipo. La numeración del conversor reductor comercial utilizado es XL4015 (XLSEMI, 2017).

\section{- Conexión y desconexión de módulos solares}

En los módulos fotovoltaicos se desea evitar la circulación de corriente inversa, es decir del prototipo a los módulos fotovoltaicos, por lo que es necesario desconectar los módulos del regulador de carga cuando no generen energía eléctrica. La utilización de diodos Schottky no es una opción válida debido a que existe una caída de voltaje de $1 \mathrm{~V}$ cuando circula $0,2 \mathrm{~A}$ por cada módulo solar (VISHAY, 2017). Por lo que se implementó un arreglo optoacoplador - MOSFET que reduce dicha caída de voltaje a $0,4 \mathrm{~V}$ y realiza la conexión y desconexión de paneles fotovoltaicos por medio del elemento inteligente del prototipo.

El microcontrolador conecta y desconecta los módulos solares, por lo que éste se informa el voltaje existente en los módulos fotovoltaicos por medio de un divisor de voltaje resistivo que reduce el voltaje al nivel de operación de la tarjeta de desarrollo y ésta lo recibe por medio de su conversor ADC. Se utiliza un potenciómetro de $100 \mathrm{k} \Omega$ y el valor de las resistencias se obtiene a través de la Ecuación (15) (Velasco, Soler, \& Botina, 2016).

$$
V_{\text {salida }}=V_{\text {entrada }} \times \frac{R_{2}}{R_{1}+R_{2}}
$$

$V_{\text {salida }}$ : tensión de salida $(\mathrm{V}), V_{\text {entrada }}$ : tensión de entrada $(\mathrm{V})$, $R_{1}$ y $R_{2}$ : resistencias $(\Omega)$.

$$
\begin{gathered}
\frac{3 V}{6 V}=\frac{R_{2}}{100 k \Omega} \\
R_{2}=50 \mathrm{k} \Omega ; R_{1}=50 \mathrm{k} \Omega
\end{gathered}
$$

En la Figura 3, se observa el arreglo entre el optoacoplador y el MOSFET.

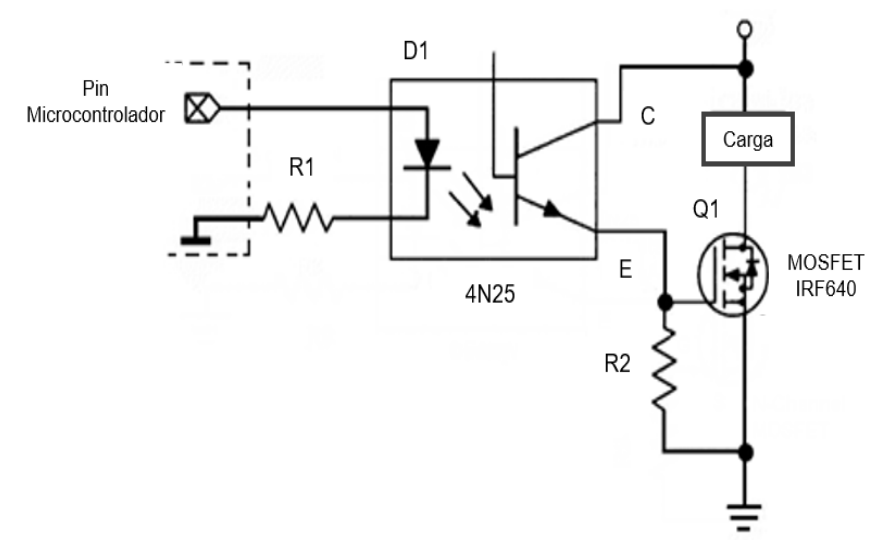

Figura 3. Arreglo optoacoplador-MOSFET

El optoacoplador evita problemas de referencia de la señal proveniente del microcontrolador cuando se conecta y desconecta los módulos solares, con la Ecuación (16) se dimensiona la resistencia que limita la corriente del LED en la entrada del optoacoplador, para que ésta sea de $2 \times 10^{-3} \mathrm{~A}$ (Toshiba, 2018).

$$
R 1=\frac{\left(V_{\text {entrada }}-V_{f}\right)}{I_{f}}
$$


$R 1$ : resistencia de entrada para limitar corriente $(\Omega), V_{\text {entrada }}$ : voltaje de entrada $(\mathrm{V}), V_{f}$ : voltaje del indicador LED $(\mathrm{V}), I_{f}$ : corriente en el LED (A).

$$
R 1=\frac{(3,1-1,05) V}{2 m A}=1025 \Omega \Rightarrow \mathrm{R} 1=1200 \Omega
$$

Por lo que se necesita una resistencia limitadora de $1200 \Omega$ para que la corriente en el LED sea de $2 \mathrm{~mA}$.

Por otra parte, para dimensionar la resistencia de polarización del MOSFET se calcula la corriente del colector en los terminales de salida del optoacoplador con la Ecuación (17) (Toshiba, 2018).

$$
C T R=\frac{I_{C}}{I_{f}} \times 100 \%
$$

$C T R$ : factor que indica la correspondencia entre corriente de salida de colector y la corriente de entrada al LED, $I_{C}$ : corriente de colector (A), $I_{f}$ : corriente en el LED (A).

$$
I_{C}=0,3 \times 2 m A=0,6 m A
$$

Para la obtención de la resistencia que polarizará al MOSFET se tiene la Ecuación (18) (Toshiba, 2018).

$$
R 2=\frac{V_{C C}-V_{C E(s a t)}}{I_{C}}
$$

$R 2$ : resistencia de polarización del MOSFET $\Omega, V_{C C}$ : voltaje de cada módulo fotovoltaico (V), $V_{C E(s a t)}$ : voltaje de saturación entre los terminales colector y emisor $(\mathrm{V})$.

$$
R 2=\frac{5-0,4}{0,6 m A}=7,6 K \Omega \Rightarrow R 2=8,2 \mathrm{k} \Omega
$$

Por lo que se necesita una resistencia de $8,2 \mathrm{k} \Omega$ para la polarización del MOSFET.

Por último, los subsistemas diseñados se los integra dentro de una placa de cobre, constituyendo así el prototipo final o sensor autónomo, como lo muestra la Figura 4.

Además, se le coloca una carcasa de protección al prototipo con salida exterior para los conectores de medición de corriente y para la antena de comunicación inalámbrica, a fin de que la comunicación inalámbrica tenga un mayor alcance, como lo muestra en la Figura 5.

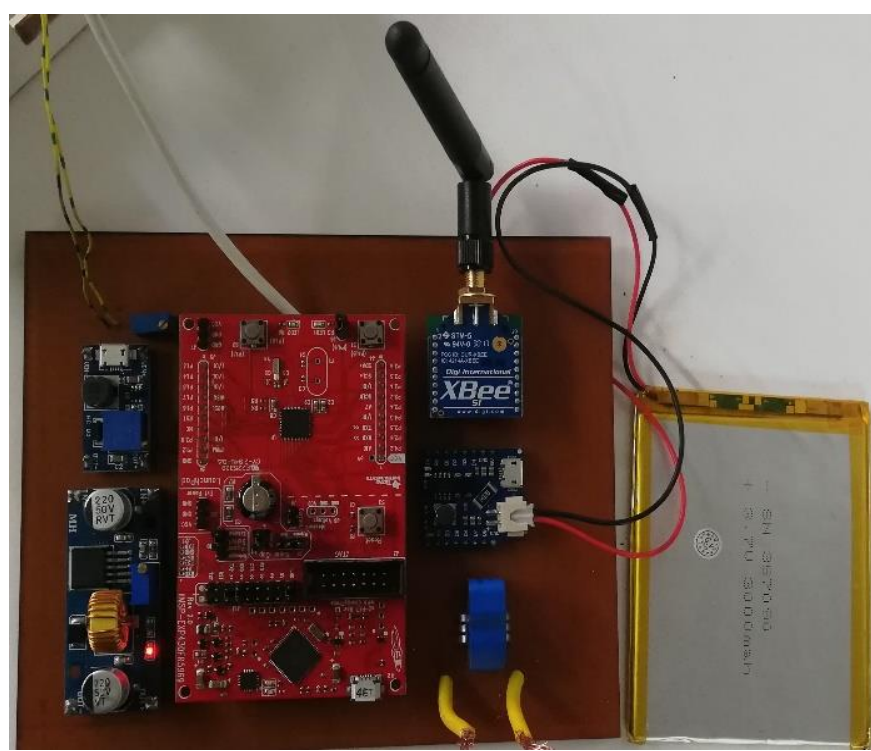

Figura 4. Vista superior de la placa base

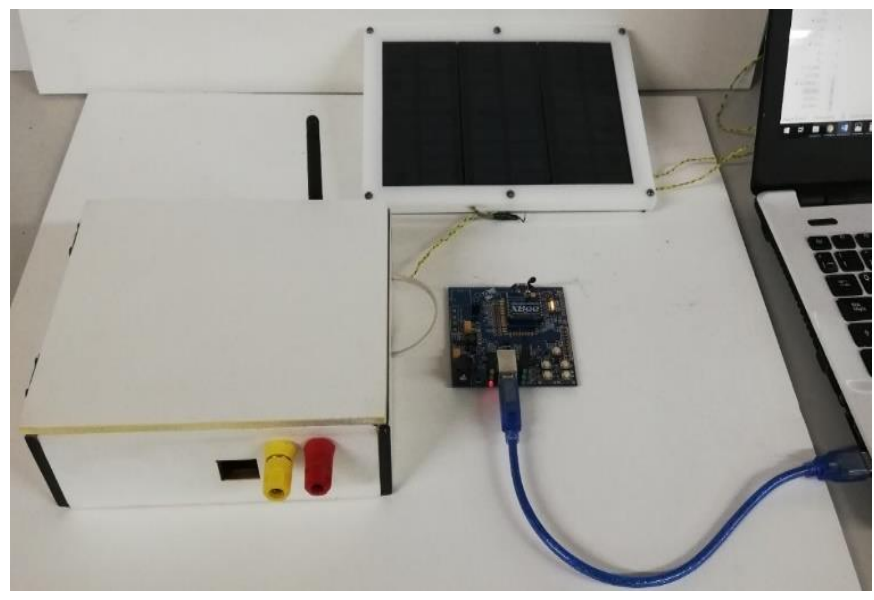

Figura 5. Implementación del sensor autónomo

\section{PRUEBAS Y RESULTADOS}

Se realizan pruebas de funcionamiento para comprobar que el sensor autónomo opere de manera adecuada y, a continuación, se detallan los resultados obtenidos.

\subsection{Prueba de almacenamiento de energía}

En un día seleccionado al azar se mide voltaje y corriente para verificar la generación de energía eléctrica de los módulos mientras alimentan al prototipo. Se implementó un circuito de adquisición de datos de ambas señales eléctricas con sus respectivos acondicionamientos de voltaje para que no dañen al microcontrolador. Esta prueba fue realizada durante 24 horas continuas para determinar las horas en las que se tiene una mayor generación energética y verificar si es la suficiente para cargar la batería implementada. La adquisición de los datos de las señales eléctricas se la realiza mediante un microcontrolador, el cual envía esta información al software LabVIEW mediante comunicación serial. Los datos adquiridos se almacenan en Excel para su posterior representación, como se observa en las Figuras 6 y 7. 


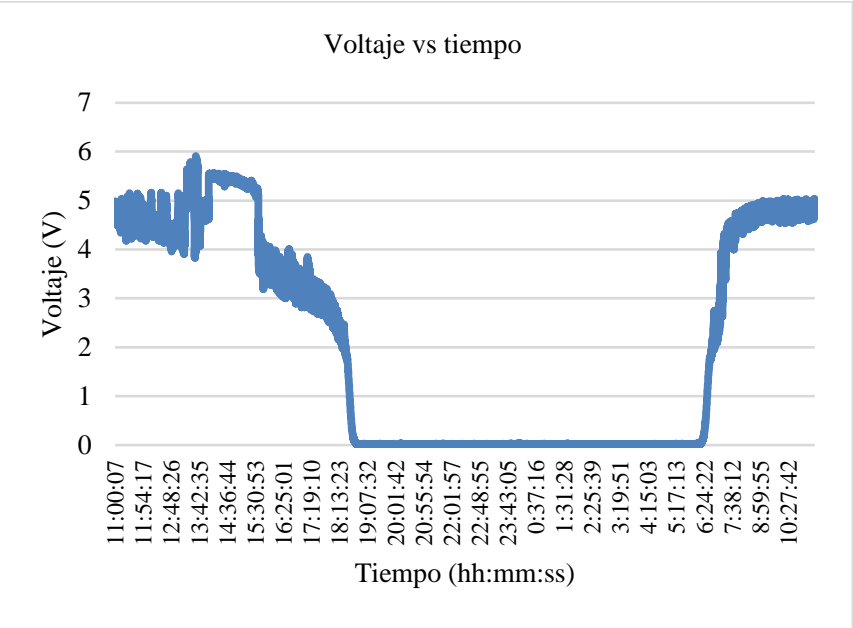

Figura 6. Gráfica voltaje vs tiempo de los módulos solares

Como lo muestra la Figura 6, el voltaje que se tiene entre las 8:00 am hasta las 04:00 pm es mayor al voltaje de carga de la batería, que es de $4 \mathrm{~V}$, lo cual permite se realice la carga del acumulador de energía.

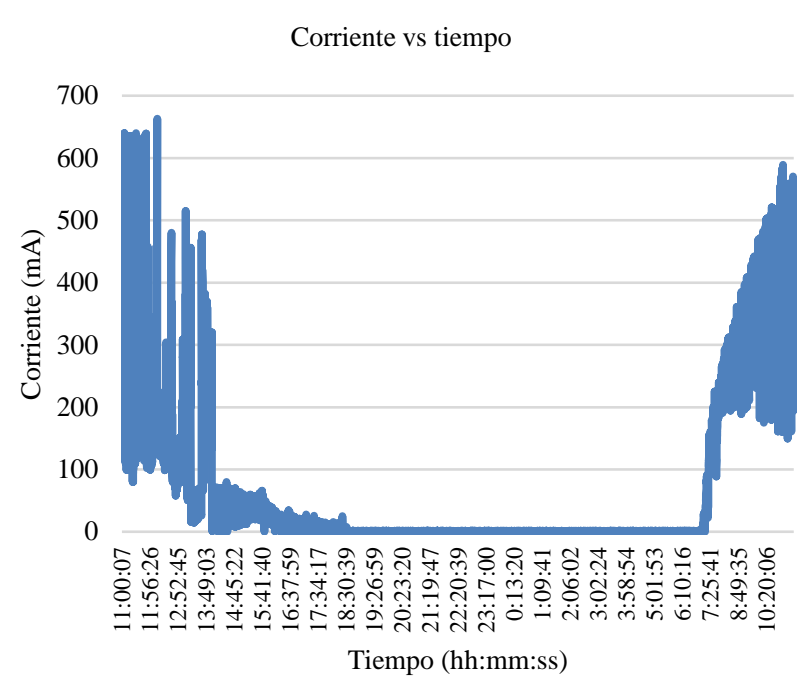

Figura 7. Gráfica corriente vs tiempo de los módulos solares

En la Figura 7, se puede observar que la corriente existente entre las 8:00 am hasta las 02:00 pm es mayor a $300 \mathrm{~mA}$, por lo que se tiene el tiempo suficiente y un valor de corriente adecuado para cargar la batería en su totalidad en caso de que se encuentre descargada.

\subsection{Prueba de consumo energético del sensor diseñado}

Se comprueba el bajo consumo de energía del prototipo mediante una herramienta llamada Energy Trace que se encuentra dentro del programa Code Composer Studio, dicha herramienta analiza el código del microcontrolador por 30 segundos y muestra la potencia consumida por el microcontrolador considerando todos los elementos conectados a sus periféricos. Se realizan dos pruebas dentro de esta herramienta, la primera, es cuando el prototipo se encuentra transmitiendo información y, la segunda, cuando no transmite información y entra en un estado de bajo consumo de potencia. Como resultado de dichas pruebas las Figuras 8 y Figura 9 muestran el consumo de potencia en función del tiempo para cada caso.

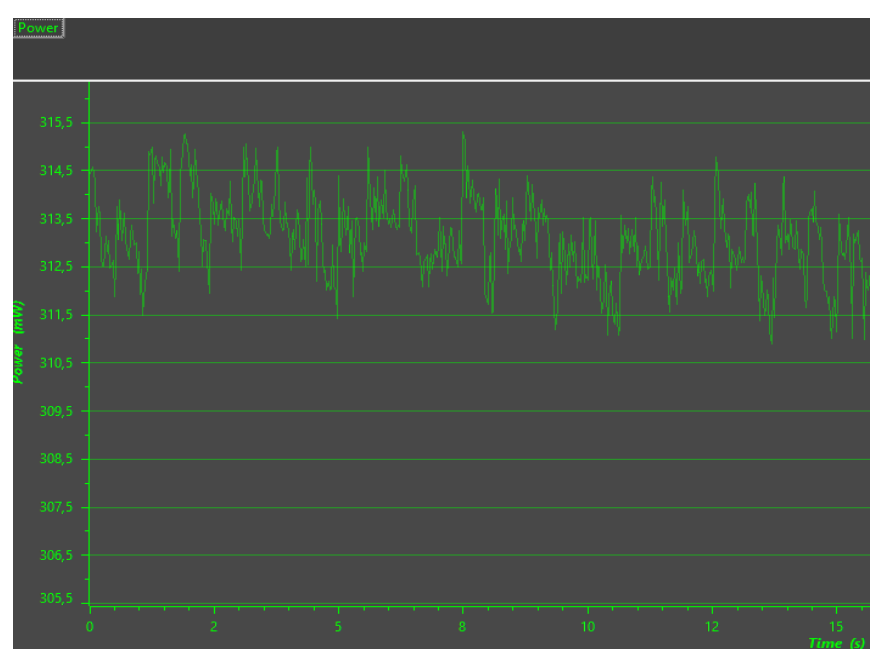

Figura 8. Consumo de potencia del prototipo; transmitiendo información

En la Figura 8, se puede observar el consumo de potencia que tiene el sensor autónomo cuando se encuentra transmitiendo información, este valor es de alrededor de $313 \mathrm{~mW}$.

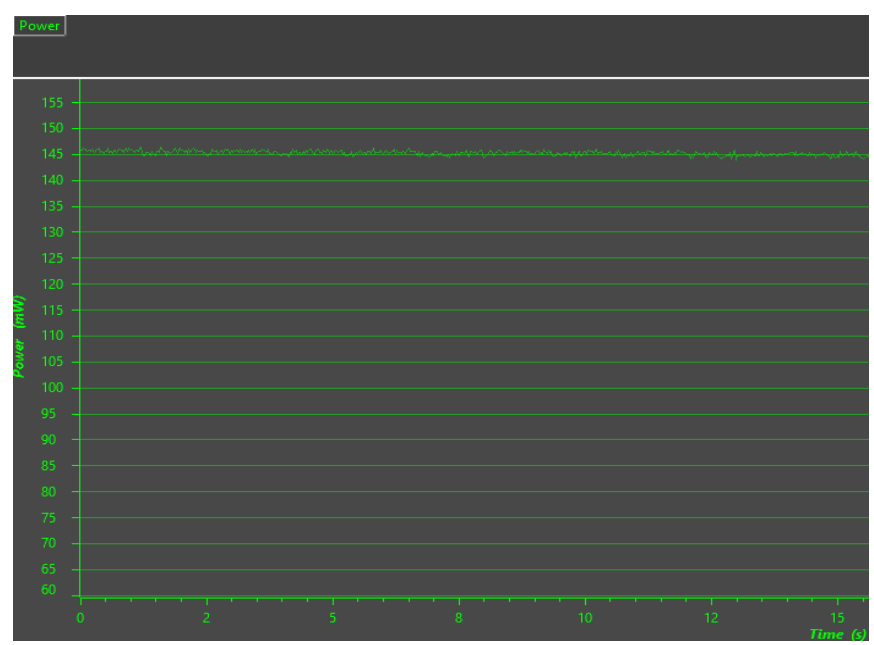

Figura 9. Consumo de potencia del prototipo; no transmitiendo información

El momento en que el prototipo no se encuentra transmitiendo información, el consumo de potencia disminuye hasta un valor constante de $145 \mathrm{~mW}$ como se muestra en la Figura 9.

Se ha comprobado que el sensor autónomo consume menor cantidad de energía cuando se encuentra en un estado de bajo consumo de potencia al no estar enviando constantemente información.

Otra funcionalidad de EnergyTrace es que dicha herramienta muestra el tiempo de autonomía que la batería puede brindar al prototipo y este valor fue de aproximadamente dos días, tal como se había propuesto en el diseño. 


\subsection{Prueba con motor de inducción}

Una forma de comprobar el funcionamiento del prototipo, ante una perturbación, es realizar una prueba durante la operación de un motor de inducción trifásico, conectando el sensor autónomo a una de sus fases. La prueba consiste en encender el motor de inducción hasta llegar a su estado de operación normal, luego, indicar al sensor autónomo para que adquiera los valores de corriente y con ellos calcule un valor rms aproximado para que, finalmente, determine los valores picos de corriente y defina éstos como los límites entre el estado de transmisión y el de no transmisión de información. Las mediciones en operación normal pueden ser visualizadas mediante comunicación serial como se muestra en la Tabla 7.

\begin{tabular}{lc}
\multicolumn{2}{c}{ Tabla 7. Mediciones de corriente en operación normal } \\
\hline \multicolumn{1}{c}{ Datos de corriente de operación normal medidos } \\
\hline Irms: & $1.20 \mathrm{~A}$ \\
Valor pico superior: & $1.76 \mathrm{~A}$ \\
Valor pico inferior: & $-1.76 \mathrm{~A}$ \\
\hline
\end{tabular}

Para continuar con la prueba, se apaga y se vuelve a encender al motor, al tener una corriente de arranque con valor superior a la corriente en operación normal, el prototipo comienza a transmitir los datos de corriente hasta finalizar el estado transitorio y llegar a su operación normal.

En la Figura 10, se observan los resultados transmitidos por el prototipo durante el arranque del motor, los cuales fueron almacenados en LabVIEW y exportados a un archivo Excel para su representación.

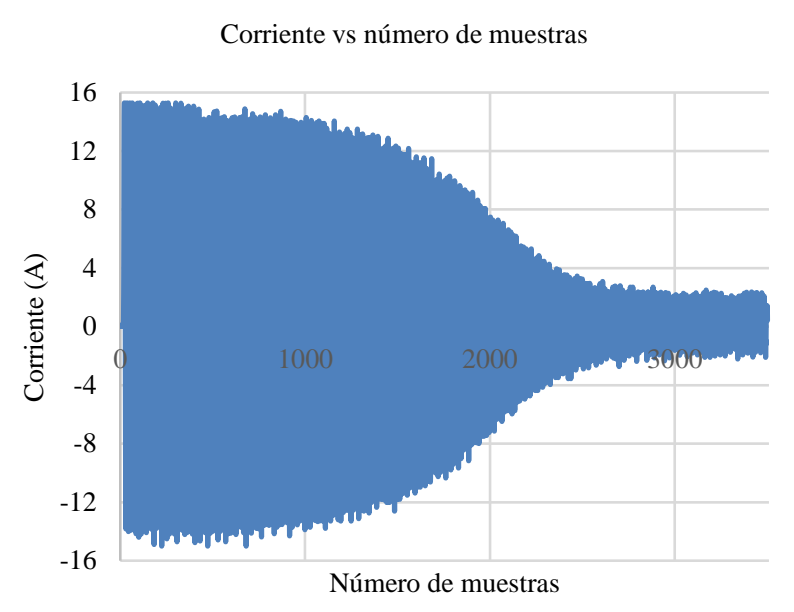

Figura 10. Corriente de arranque motor trifásico de inducción - Excel

Considerando que el arranque del motor tiene un transitorio con una duración aproximada de 3 segundos y considerando que se registran 20 muestras por ciclo, en una red de 60 ciclos por segundo, se tienen 3600 datos transmitidos por el prototipo durante el arranque del motor. Luego del arranque del motor la corriente vuelve a estar por debajo de los límites normales de operación y el sensor autónomo deja de transmitir información, entrando en modo de bajo consumo.

El motor utilizado tiene las especificaciones técnicas que se muestran en la Tabla 8.
Tabla 8. Datos de placa del motor de inducción utilizado

\begin{tabular}{cccccc}
\hline \multicolumn{5}{c}{ Bruxelles - CETEL } \\
\hline Tipo & S611 & Número & 17 & f & $60 \mathrm{~Hz}$ \\
V & $220-380 V$ & Corriente & $7-4$ A & T.min & 1800 \\
CV & 2 & Fases & 3 & $\cos \boldsymbol{\varphi}$ & - \\
\hline
\end{tabular}

\subsection{Prueba del sensor prototipo aplicada a un transformador}

Se realiza la prueba de cortocircuito en un transformador monofásico. Inicialmente se le conecta alimentación de $120 \mathrm{~V}$ y carga de $33 \Omega$ con factor de potencia unitario en cada bobinado $\mathrm{y}$, mediante un conmutador, se produce un cortocircuito al devanado secundario del transformador monofásico que, a su vez, induce elevadas corrientes en el bobinado primario, lugar de conexión del sensor autónomo. Para la protección de las bobinas se utilizó un fusible de 5A. Esta prueba comprueba el correcto funcionamiento del prototipo ante perturbaciones con una elevada corriente y un transitorio de corta duración debido a que los fusibles actúan en el momento que su filamento se funde. Los datos fueron almacenados y graficados en un archivo Excel, como se muestra en la Figura 11.

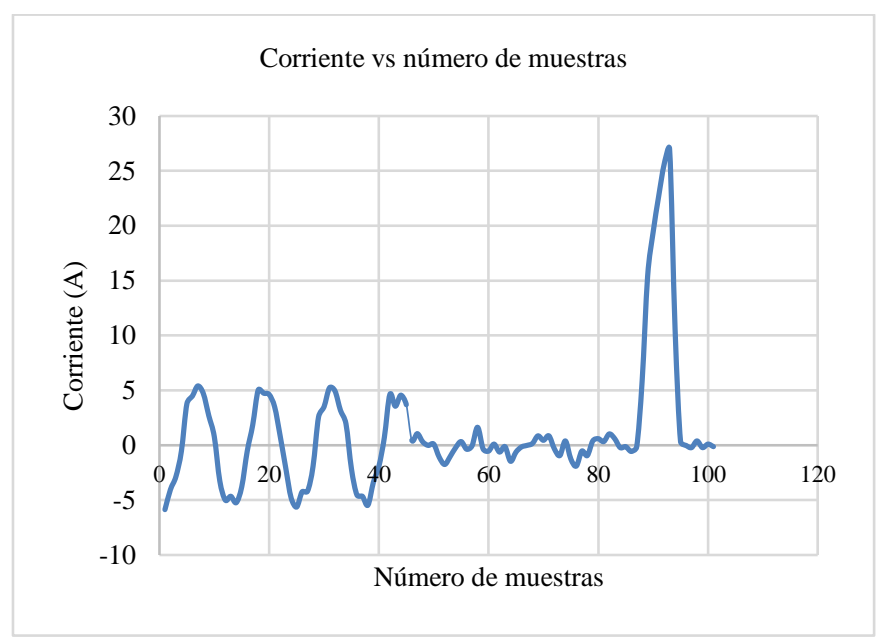

Figura 11. Corriente de cortocircuito en transformador - Excel

Los resultados obtenidos comprueban el funcionamiento del sensor autónomo, ya que, al detectar una señal de corriente mayor a los límites establecidos para la transmisión, el prototipo transmite la información y como se observa en la Figura 11, la corriente llega a valores de 28 A pico, donde el fusible se funde y la corriente se hace cero.

\subsection{Prueba de alcance de comunicación}

En esta prueba se transmite 100 conglomerados de datos enviados desde el emisor hacia el receptor, a distintas distancias de separación, para determinar la máxima distancia a la cual los módulos se intercomunican; para este caso, se mide y analiza el indicador de la fuerza con que llega la señal y, para asegurar una buena calidad en la señal, este valor puede llegar hasta $-70 \mathrm{dBm}$ (Núñez y col. 2017). Para la prueba los módulos XBee tienen una directa línea de visión y la prueba se realiza en exteriores e interiores. 
El programa XCTU realiza pruebas de rango en medio de los módulos de comunicación y al final permite obtener como resultado la potencia de la señal y el porcentaje exitoso en el envío y recepción a determinadas distancias, estos valores se indican en la Tabla 9.

Tabla 9. Pruebas de rango de comunicación inalámbrica

\begin{tabular}{ccccc}
\hline \multicolumn{5}{c}{ Pruebas realizadas en exteriores } \\
\hline $\begin{array}{c}\text { Distancia } \\
\text { entre } \\
\text { módulos } \\
{[\mathbf{m}]}\end{array}$ & $\begin{array}{c}\text { Datos } \\
\text { enviados }\end{array}$ & $\begin{array}{c}\text { Datos } \\
\text { recibidos }\end{array}$ & $\begin{array}{c}\text { Tasa de envío y } \\
\text { recepción [\%] }\end{array}$ & $\begin{array}{c}\text { Potencia } \\
\text { en la señal } \\
\text { [dBm] }\end{array}$ \\
\hline 10 & 100 & 100 & 100 & -57 \\
20 & 100 & 100 & 100 & -60 \\
30 & 100 & 100 & 100 & -62 \\
40 & 100 & 97 & 97 & -66 \\
50 & 100 & 96 & 96 & -68 \\
60 & 100 & 92 & 92 & -70 \\
70 & 100 & 58 & 58 & -75 \\
\hline \multicolumn{5}{c}{ Pruebas realizadas en interiores } \\
\hline 10 & 100 & 100 & 100 & -50 \\
20 & 100 & 100 & 100 & -57 \\
30 & 100 & 99 & 99 & -59 \\
40 & 100 & 99 & 99 & -63 \\
50 & 100 & 66 & 66 & -75 \\
\hline
\end{tabular}

Como resultados de la prueba de comunicación, sin conexión alámbrica, se tiene que en campo abierto existen un alcance de $60 \mathrm{~m}$ con una buena calidad en la señal y una tasa de carga y descarga exitosa superior al $92 \%$, por otra parte, en instalaciones interiores donde existen elementos que obstaculizan la comunicación, la distancia de alcance disminuyó a $40 \mathrm{~m}$.

\section{CONCLUSIONES}

De los distintos recursos energéticos medio ambientales, se definió el uso de energía solar como fuente de energía para el sensor inalámbrico autónomo debido a que esta tecnología se encuentra más desarrollada, tiene un bajo costo de implementación y en el mercado se dispone de gran variedad de los elementos que la constituyen en comparación con otros tipos de energía.

En la operación del prototipo se logró un muy bajo consumo de energía, igual a 4,67 Wh/día, mediante dos dispositivos fundamentales que son: la tarjeta MSP430-FR5969 y los módulos de comunicación XBee S1, priorizando que estos últimos elementos trabajen en modo de bajo consumo cuando no sea necesaria la transmisión de información.

En el prototipo se logró implementar un algoritmo de procesamiento de datos que permite determinar si los valores de corriente del sistema de prueba se mantienen o salen del rango normal de operación y que a su vez ayuda al ultra bajo consumo de energía del prototipo. Además, con el fin de lograr la detección de perturbaciones muy rápidas en la señal medida, se estableció un tiempo de muestreo, de 20 muestras por ciclo, que asegura la obtención de una gran cantidad de datos para cada posible evento atípico presente en un sistema eléctrico, tal como los muestran los resultados de las pruebas realizadas en este artículo.
Se determinó las distancias máximas para la comunicación entre los módulos XBee S1, obteniéndose distancias mayores para cuando se trabaja en exteriores, lo que permite que el sensor autónomo, que contiene al emisor, pueda colocarse hasta a $60 \mathrm{~m}$ con línea de visión directa al receptor, ahorrando un costo económico en la implementación del cableado para la transmisión de las señales. Al trabajar en interiores, el alcance de comunicación se redujo a $40 \mathrm{~m}$ debido a paredes y objetos que disminuyen la potencia de la señal.

\section{AGRADECIMIENTO}

El presente artículo se desarrolló gracias al apoyo del Dr. Sorin Melinte de la Université Catholique de Louvain (UCL) Bélgica.

\section{REFERENCIAS}

Abella, M. A. (2012). Sistemas Fotovoltaicos. Madrid, España: Centro de Investigaciones, Medioambientales y Tecnológicas, 3-6.

AEROSEMI. (2015). MT3608. Pekin, China: AEROSEMI, 1-5.

Agencia de Regulación y Control de Electricidad. (2019). Pliego tarifario para las empresas eléctricas de distribución codificado. Quito, Ecuador: ARCONEL, 20.

Alonso Lorenzo, J. A. (2016). SUNFIELDS EUROPE (Manual para el cálculo de paneles fotovoltáicos). Obtenido de: https://www.sfesolar.com/baterias-solares/manual-calculo/. (Mayo, 2019).

Caina Aysabucha, Darwin R. et al. (2019). Flexible SiNWs-based thermoelectric generators for healthcare monitoring, Conference E-MRS 2019 Spring Meeting, Nice-France.

Catalán López, J. (2013). Energy Harvesting: Estado del arte y diseño de un sensor de temperatura inalámbrico alimentado por energìa fotoeléctrica con sistema de alimentación de apoyo por radiofrecuencia, Trabajo de Maestría, Zaragoza: Universidad de Zaragoza, 10-50.

CIRCE. (2015). Energìa Fotovoltaica conectada a la red. Capacitación para análisis y regulatorio de la inmersión de generación no convencional en el SIN del Ecuador. Zaragoza.

DIGI. (2018). XBee/XBee-PRO S1 802.15.4 (Legacy) RF MODULES. Obtenido de: https://www.digi.com/resources/documentation/digidocs/PDFs/90 000982.pdf. (Febrero, 2019).

IXYS. (2018). IXOLAR High Efficiency SolarMD. Obtenido de: http://ixapps.ixys.com/DataSheet/SLMD600H10L_Nov16.pdf. (Mayo, 2019).

J\&A. (2012). Lithium-ion Polymer Battery Specification. Obtenido de: https://www.olimex.com/Products/Power/BATTERYLIPO250mAh/resources/JA602025P-Spec-Data-Sheet-3.7V250mAh--170116.pdf. (Enero, 2019).

LEM. (2017). Current Transducer LTS 15-NP. Obtenido de: https://www.lem.com/sites/default/files/products_datasheets/lts_1 5-np.pdf. (Enero, 2019).

Mijarez, R. (2014). Electrónica. Ciudad de México, México: Patria, 120-126.

Núñez Pérez, J. C., Bonilla Rodriguez, A., \& Calvillo Téllez, A. (2017). Estimación del alcance de radiotransmisores Xbee. Trabajo de Titulación, Baja California: Instituto Politécnico Nacional, 15-19.

Ochoa Méndez, A. E. (2013). Estudio para determinar las pérdidas de energía del alimentador 124 pertenecientes al sistema de distribución de la empresa eléctrica Azogues. Trabajo de Titulacion, Cuenca: Universidad Politécnica Nacional, 15-21.

Ramirez Castaño, J. S. (2014). Protección de Sitemas Eléctricos . Manizales, Colombia: Universidad Nacional de Colombia, 65,66.

Rustom J., A. (2012). Estadística descriptiva, probabilidad e inferencia. Santiago de Chile, Chile: Universidad de Chile, 30-35.

Texas Instruments. (2015). MSP430FR5969 LaunchPad Development Kit. Obtenido de: http://www.ti.com/tool/MSP-EXP430FR5969. (Febrero, 2019).

Texas Instruments. (2017). MSP430FR59XX User's Guide. Obtenido de: http://www.ti.com/lit/ug/slau367o/slau367o.pdf. (Febrero, 2019).

Toshiba. (2018). Basic characteristics and application circuit design of IC couplers, Tokio, Japón: Toshiba, 8-13. 
Velasco, A., Soler, J., \& Botina, O. (2016). Divisores de tensión y corriente. Trabajo de Titulación, Medellín: Universidad Nacional de Colombia, 3-10.

VISHAY. (2003). 1N4728A to 1N4764A. Obtenido de: https://www.vishay.com/docs/85816/1n4728a.pdf. (Febrero, 2019).

VISHAY. (2017). BAT42, BAT43 Small Signal Schottky Diode. Obtenido de: https://www.vishay.com/docs/85660/bat42.pdf. (Mayo, 2019).

XLSEMI. (2017). XL4015 Buck DC to DC Converter. Obtenido de: http://www.xlsemi.com/datasheet/XL4015\%20datasheet.pdf. (Mayo, 2019).

\section{BIOGRAFÍAS}

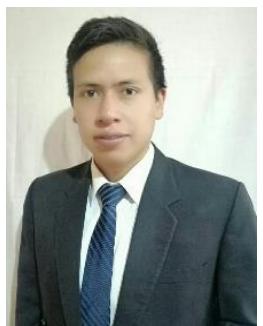

Andrés Marcelo Barahona Correa. Nació en Quito, Ecuador en 1995. Recibió el título de Ingeniero en Electrónica y Control de la Escuela Politécnica Nacional en 2019. Actualmente se desempeña como encargado de laboratorio en la empresa Optimovilidad S.A.

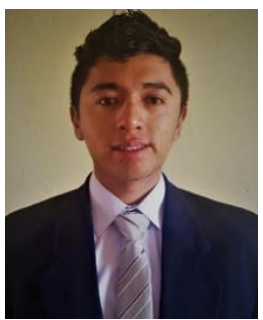

Jaime Oswaldo Salazar Cajamarca. Nació en Quito, Ecuador en 1995. Recibió el título de Ingeniero Eléctrico de la Escuela Politécnica Nacional en 2019. Actualmente se desempeña como Supervisor Eléctrico en el proyecto "Rehabilitación integral emergente del sistema de bombeo del sistema Papallacta Integrado, en las estaciones Elevadora, Booster I y Booster II del sistema Papallacta Integrado".

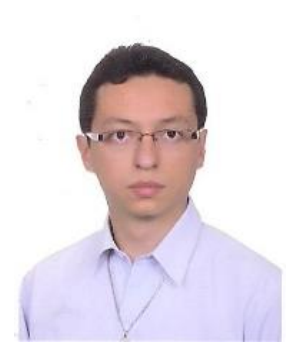

Mauricio Santiago Soria Colina. Nació en Ambato, Ecuador en 1991. Recibió el título de Ingeniero Eléctrico de la Escuela Politécnica Nacional en 2016. En 2020, obtuvo el título de Magíster en Electricidad mención Redes Eléctricas Inteligentes en la Escuela Politécnica Nacional. Actualmente se desempeña como Técnico Docente en la institución donde cursó sus estudios superiores. Su campo de investigación está relacionado con el desarrollo de Sistemas Eléctricos Inteligentes.

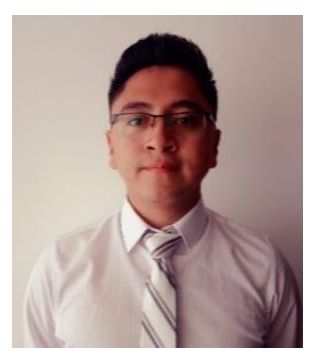

Edison Gabriel Novoa Guamán. Nació en Quito, Ecuador en 1992. Recibió el título de Ingeniero Eléctrico de la Escuela Politécnica Nacional en 2015. En 2021, obtuvo el título de Magíster en Ingeniería Eléctrica en Distribución en la Escuela Politécnica Nacional. Trabajó como técnico eléctrico en Help Energy Consultores S.A., la Empresa Eléctrica Quito y actualmente se desempeña como Técnico Docente en la institución donde cursó sus estudios superiores. Su campo de interés es el estudio de los Sistemas Eléctricos de Distribución de Energía Eléctrica.

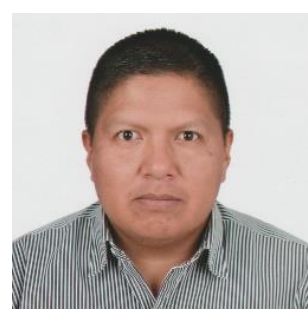

Darwin Rodolfo Caina Aysabucha. Nació en Ambato (Ecuador). Ingeniero en Electrónica y Telecomunicaciones de la Escuela Politécnica Nacional. Máster de Ciencias en Tecnologías de la Información de la Hochschule Mannheim (Alemania). Cursa estudios de Doctorado en la Université catholique de Louvain con el apoyo de ARES (Académie de Recherche et d'Enseignement Supérieur) de Bélgica. Profesor titular en la Facultad de Ciencias de la Universidad Central del Ecuador. Sus ramas de interés e investigación Nanoelectrónica, Microelectrónica, Antenas, Internet de las cosas (IoT) para aplicaciones de salud y agricultura.

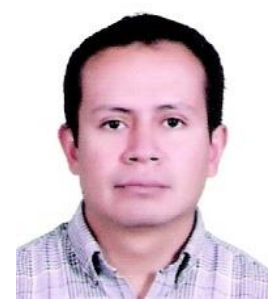

Fabián Ernesto Pérez Yauli, nació en Ambato- Ecuador. Obtuvo el título de Ingeniero Eléctrico en la Escuela Politécnica Nacional, Quito-Ecuador en 2004. En 2012 obtuvo el grado de Doctor en Ingeniería Eléctrica en la Universidad Nacional de San Juan, San JuanArgentina, con una beca otorgada por el Servicio Alemán de Intercambio Académico (DAAD). Actualmente es profesor titular a tiempo completo en la Escuela Politécnica Nacional. Su rama de investigación incluye protecciones de sistemas de potencia y procesamiento de señales. 